\title{
Current Advances for Aesthetic Improvement in Breast Reconstruction: Mimicking the Augmented Breast
}

\author{
Katherine A Rodby ${ }^{1}$, Karina P Quinn ${ }^{1}$, Babak Mehrara ${ }^{2}$ and Anuja K Antony ${ }^{1^{*}}$ \\ ${ }^{1}$ Division of Plastic, Reconstructive and Cosmetic Surgery, University of Illinois at Chicago, Chicago, IL, USA \\ ${ }^{2}$ Memorial Sloan-Kettering Cancer Center, New York, NY, USA
}

"Corresponding author: Anuja K Antony, Director of Breast Reconstruction, Division of Plastic and Reconstructive Surgery, University of Illinois at Chicago, Chicago, IL 60601, USA, Tel: 312-996-9313; Fax: 312-413-0496; E-mail: akantony@uic.edu

Rec date: Jun 11, 2014; Acc date: Jul 30, 2014; Pub date: Aug 01, 2014

Copyright: $\odot 2014$ Rodby KA et al. This is an open-access article distributed under the terms of the Creative Commons Attribution License, which permits unrestricted use, distribution, and reproduction in any medium, provided the original author and source are credited.

\begin{abstract}
Background: Women seeking implant-based breast reconstruction increasingly request a round, projecting breast with an amplified upper pole that mimics the augmented breast. Two-stage reconstruction provides the opportunity to recreate a well-defined inframammary fold (IMF) with ptosis, to modify final implant characteristics (volume upsizing, high profile, gel implants) and correct contour irregularities with lipofilling at exchange to achieve this desired outcome.
\end{abstract}

Methods/Technique: All patients completing two-stage breast reconstruction were added prospectively into a database. Patient demographics, oncologic phenotype and treatment, surgical details including IMF reconstruction, implant characteristics, and outcomes were reviewed. 2D and 3D photos and intraoperative videography were captured, further illustrating the technique.

Results: 84 two-stage breast reconstructions were completed in 50 women between 5/2010- 11/2013. Mean age at the time of mastectomy was 42.8 years and mean BMI was 30 . At the time of exchange, the majority of implants were typically upsized from the final expansion volume, with a volume increase ranging from 10-150cc (mean increase 46cc). Mean expander fill volume and final implant volume were $595 \mathrm{cc}$ and $618 \mathrm{cc}$ respectively. Fifty-nine reconstructions $(70 \%)$ included inframammary fold reinforcement. Sixty-eight $(81 \%)$ of reconstructions utilized high profile or extra-high profile implants. Selective use of fat grafting was implemented later in the series.

Conclusion: Current advances in two-stage implant based breast reconstruction are outlined to achieve upper pole fullness, anterior projection of the breast, and definition of the inframammary fold. These strategies afford reconstructive outcomes that parallel the desired aesthetic features of the augmented breast.

Keywords: Two-stage reconstruction; Inframammary fold suspension; Aesthetic breast reconstruction; Tissue expander reconstruction

\section{Introduction}

Aesthetic breast reconstruction after mastectomy impacts a woman's post-surgical confidence, sexuality, and overall well-being [1]. With increased patient awareness, expectations after breast reconstruction have increased manifold and assimilated towards that of the post-augmentation breast. The mainstay of breast reconstruction continues to be two stage tissue expander/implant (TE/I) based reconstructions, with aesthetic results hinging upon the creation of a natural breast shape with upper pole fullness, lower pole ptosis, and a well-defined inframammary fold (IMF) [2,3]. However, many surgeons fail to adequately address second stage procedures or the reconstruction process overall that can help achieve improved cosmesis $[2,3]$.

Aesthetic breast reconstruction encompasses several aspects of breast symmetry and profile, including projection, ptosis, sulcus location, and lack of skin irregularities [3]. Adequate IMF reconstruction requires precise capsulotomy placement, tissue recruitment and dermal suspension to restore the blunted or eradicated fold. While IMF re-suspension is critical to achieve a natural breast shape, additional techniques to improve upper pole fullness, with creation of a round and projecting breast may be as important to achieve a successful reconstruction in patients desiring a construct which mimics the augmented breast. The exchange procedure in two-stage reconstruction provides the opportunity to modify final implant characteristics via upsizing volume and employing high profile, silicone implants to achieve the desired outcome. This paper describes current advances in aesthetic techniques utilized in a consecutive patient series, which focuses on two- stage reconstructions to simulate an augmented breast result.

\section{Patients and Methods}

Between May 2010 and November 2013, 84 consecutive two-stage breast reconstructions were completed in 50 women at the University of Illinois at Chicago and Mount Sinai Medical Center. All patients undergoing breast reconstruction were added prospectively into a database, recording patient demographics, oncologic phenotype, adjuvant treatment, reconstructive details, tissue expander and implant characteristics, and outcomes. Patients undergoing tissue expander reconstruction were identified; however, only those patients 
who completed second stage reconstruction were included in the analysis.

Over a period of 46 months, 84 two-stage breast reconstructions were completed in 50 patients by the senior author (AKA). At the time of exchange, 59 reconstructions (70\%) included inframammary fold reinforcement. At the time of mastectomy, mean age was 42.8 years old (range 23-63) and mean BMI was 30 (range 20-42). (Table 1) Thirty four patients elected to have bilateral mastectomies while 16 patients proceeded with unilateral mastectomies. Indications for mastectomy included prophylaxis to Stage 3B breast cancer. The majority of reconstructions were immediate (73 breasts) while nine patients (11 breasts) were delayed. For non-surgical oncologic treatment, seven patients (14\%) received neoadjuvant chemotherapy, 24 patients $(48 \%)$ received adjuvant chemotherapy, and 18 patients (36\%) received radiation to the diseased breast.

\begin{tabular}{|l|l|}
\hline Patients & $\mathbf{5 0}$ \\
\hline Breast Reconstructions & 84 \\
\hline Inframammary Fold Re-suspension & $59(70 \%)$ \\
\hline Age & 42.8 \\
\hline Ethnicity & \\
\hline African American & $28(56 \%)$ \\
\hline Caucasian & $12(24 \%)$ \\
\hline Hispanic & $9(18 \%)$ \\
\hline Asian & $1(2 \%)$ \\
\hline Oncologic Treatment & \\
\hline Bilateral Mastectomy & $34(68 \%)$ \\
\hline Unilateral Mastectomy & $16(32 \%)$ \\
\hline Neoadjuvant Chemotherapy & $7(14 \%)$ \\
\hline Adjuvant Chemotherapy & $24(48 \%)$ \\
\hline Radiation Therapy & $18(36 \%)$ \\
\hline
\end{tabular}

Table 1: Patient Demographics

\section{Surgical Technique}

Immediate or delayed reconstruction was performed with Style 133MV textured tissue expanders (Allergan Inc, Irvine, CA) with inferolateral support via 1) total musculofascial coverage, 2) acellular dermal sling or 3) a latissimus flap if inadequate skin coverage due to radiation or scarring.

In the preoperative holding area, standard markings of the vertical midline, upper breast pole, and proposed and current inframammary fold position were performed with the patient standing. The expander is removed via excising the mastectomy scar, performing a limited elevation of the mastectomy flaps above the pectoralis muscle, and incising the pectoralis perpendicular to this incision, along the direction of the muscle fibers. A superior capsulotomy is completed in the supine position at the capsule reflection with re-elevation of the adherent pectoralis major. This allows the upper pole to fill with the upsized implant and drapes the skin over the superior portion of the implant, minimizing post-mastectomy contour defects.

The patient is then placed in the sitting position, and capsulotomies are carried out at the inferior, medial, and lateral aspects. Upright patient positioning is critical to promote accurate capsulotomy placement. The medial and limited lateral capsulotomies are placed 1-2 cm anterior to the reflection to support forward movement of the breast construct while maintaining the capsular edge to prevent medial and lateral implant dislodgement.

Complete IMF reconstruction requires an inferior capsule release, and the capsulotomy is placed with the intent to accurately reposition the IMF at the proposed level. It is critical to correctly position the inferior capsulotomy so that a dermal-capsule suture suspension can be performed without the need for suturing to the periosteum. Shifting the capsulotomy higher on the posterior segment effectively moves the IMF superiorly on the chest wall, while a more anterior capsulotomy shifts the dermal-capsular suspension of the IMF inferiorly. The inferior release includes parallel scoring with respect to the IMF, which continues into the fat of the anterior abdominal tissue and lower breast pocket to recruit additional breast ptosis as needed. At the inferior capsulotomy, the inferior edge of the posterior capsule is suspended to the dermis using 2-0 silk sutures. Silk sutures are placed (beginning at the midpoint and extending both medially and laterally with a slight curve) to recreate the IMF until the internal flap suspension demonstrates accurate contour and ptosis (Figure 1).

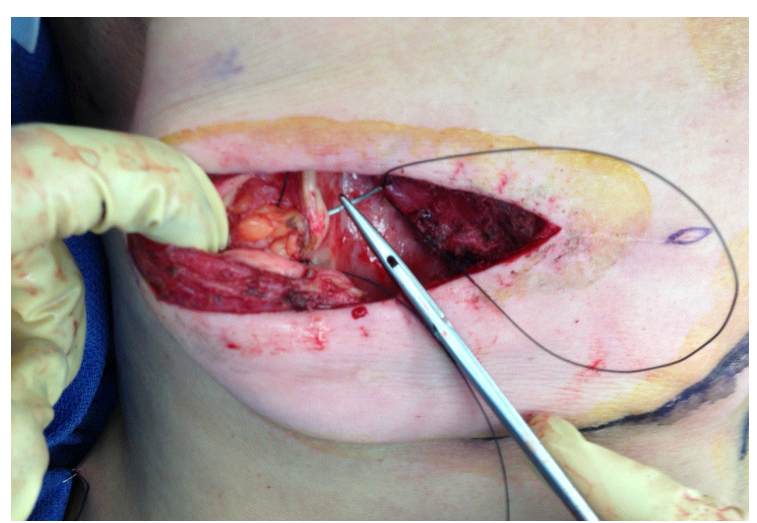

Figure 1: Lateral placement of the inframammary fold suspension sutures

A saline sizer is used to estimate the optimal volume and projection. Final placement of any additional silk sutures can be carried out until desired symmetry and definition is achieved (Figure 2). The IMF should develop a dimpled appearance initially if the sutures are placed at the appropriate dermal level (Figure 3). The chest is reprepared and the silicone implants, prepared with antibiotic irrigation and placed with minimal handling, are selected based on the intraoperatively determined saline sizer volume (upsized when compared to the tissue expander volume) to assist with breast projection and upper pole fullness. Selection of higher projection implants will improve anterior projection and upper pole fullness. Muscle, subcutaneous tissues and skin are closed in a layered closure with 2-0 and 3-0 Vicryl and 4-0 Monocryl running sutures. Dermabond is applied to all incisions and the patient is covered with fluffs and a surgical bra. The dimpling of the IMF will resolve over the ensuing 2-3 months. Fat grafting into the 
Citation: Rodby KA, Quinn KP, Mehrara B and Antony AK (2014) Current Advances for Aesthetic Improvement in Breast Reconstruction: Mimicking the Augmented Breast . Surgery Curr Res 4: 202. doi:10.4172/2161-1076.1000202

Page 3 of 6

upper pole will assist with softening post-mastectomy contour defects not improved by the superior capsulotomy and higher profile implants and can be performed at the time of exchange.

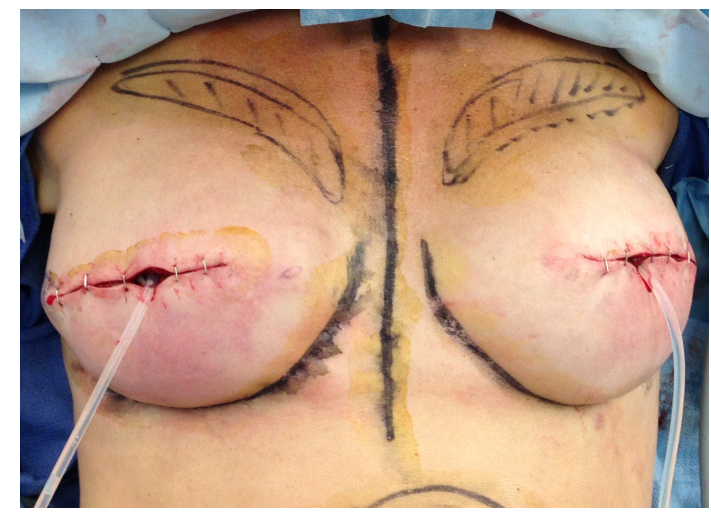

Figure 2: Use of saline sizers to evaluate the resuspended inframammary fold and determine the volume for the implant

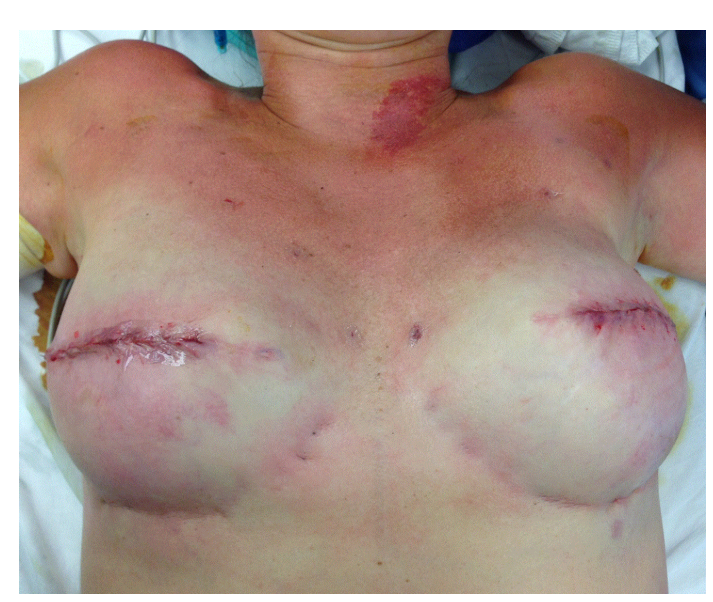

Figure 3: Dimpling of the inframammary fold at case completion

\section{Results}

Mean time for tissue expansion was 84 days and mean time to exchange was 130 days. Mean mastectomy volume was $718 \mathrm{~g}$, while mean expander fill volume and implant volume were $595 \mathrm{cc}$ and $618 \mathrm{cc}$ respectively. Sixty-six reconstructions (79\%) had acellullar dermal matrix used at the first stage of reconstruction, while 14 reconstructions (17\%) required latissimus flaps for additional tissue coverage. At the second stage, implants were typically upsized compared to the expander volume, with a mean volume increase of $47 \mathrm{cc}$ (range 10-155cc) (Table 2). Irradiated patients and postlatissimus patients were routinely over-filled during expansion to counter post-radiation skin changes and correct soft-tissue loss or scarring. These patients $(n=25)$ were excluded from volume calculations as flap volume and differences in expansion techniques in the setting of radiation could not be accounted for when comparing final implant volume to filled expansion volume.

\begin{tabular}{|l|l|}
\hline Breast Volume at Each Stage (average) & \\
\hline Mastectomy Volume & $718 \mathrm{cc}$ \\
\hline Tissue Expander Fill Volume & $595 \mathrm{cc}$ \\
\hline Implant Volume & $618 \mathrm{cc}$ \\
\hline Tissue Coverage & \\
\hline Acelluar Dermal Matrix & $66(79 \%)$ \\
\hline Latissimus flaps & $14(17 \%)$ \\
\hline Implant Styles used for Reconstruction & \\
\hline Style 45 & $28(33 \%)$ \\
\hline Style 20 & $40(48 \%)$ \\
\hline Style 15 & $8(10 \%)$ \\
\hline Style 10 & $8(10 \%)$ \\
\hline Associated Procedures & \\
\hline Contralateral augmentation & $7(14 \%)$ \\
\hline Contralateral reduction or mastopexy & $9(18 \%)$ \\
\hline Lipofilling & $21(25 \%)$ \\
\hline
\end{tabular}

Table 2: Reconstruction Demographics

In this study, silicone implants were placed exclusively at the second-stage procedure. High profile Style 20 and extra-high profile Style 45 silicone implants (Allergan Inc, Irvine, CA) were the most commonly used implants in $81 \%$ of reconstructions, $40(48 \%)$ and 28 (33\%) respectively. Other styles incorporated included Style 15, moderate plus profile, and Style 10, moderate profile in eight breasts (10\%) each.

Aesthetic outcomes were documented by two-dimensional and three-dimensional photography (Figures 4-6). Patients were followed for a mean of 21 months (range 4-48mo). Fifteen contralateral breast procedures were completed for symmetry, with seven augmentations and eight breast reductions or mastopexies. Post-reconstruction aesthetic procedures included lipofilling in 21 reconstructions, six scar revisions, and two cases of contouring liposuction. Three complications occurred during the second stage including two seromas (one resolved with drainage and one resulted in dehiscence requiring revision) and one hematoma necessitating evacuation and implant exchange. Capsular contracture requiring reoperation was documented in five reconstructions $(10 \%)$, with four due to postradiation changes. They were corrected with conversion to free flap reconstruction $(n=1)$, latissimus flap $(n=1)$, or acellular dermal matrix and revision $(n=3)$. No patients have required revision of the IMF after reconstruction.

\section{Discussion}

Over the past decade, the rates of post-mastectomy breast reconstruction continue to rise, improving body image and quality of life [4]. Considering the recent trend towards implant-based reconstruction, non-autologus reconstruction provides several challenges to achieve good cosmetic results $[5,6]$. Aesthetic results are based on the essential features of a natural breast, including size, 
Citation: Rodby KA, Quinn KP, Mehrara B and Antony AK (2014) Current Advances for Aesthetic Improvement in Breast Reconstruction: Mimicking the Augmented Breast . Surgery Curr Res 4: 202. doi:10.4172/2161-1076.1000202

Page 4 of 6

projection, upper pole contouring, lower pole fullness, ptosis, fold location, and symmetry [2,3,7]. Attaining superior cosmesis bears impact, as post-reconstruction analysis has linked patients' aesthetic scores and symmetry with patient satisfaction [7]. With recent advances in breast cancer reconstruction, expectations have also increased and paralleled that of the augmented breast. Achieving these aesthetic results typically focus on the creation of upper pole fullness, optimal projection, lower pole ptosis, and a well-defined IMF.

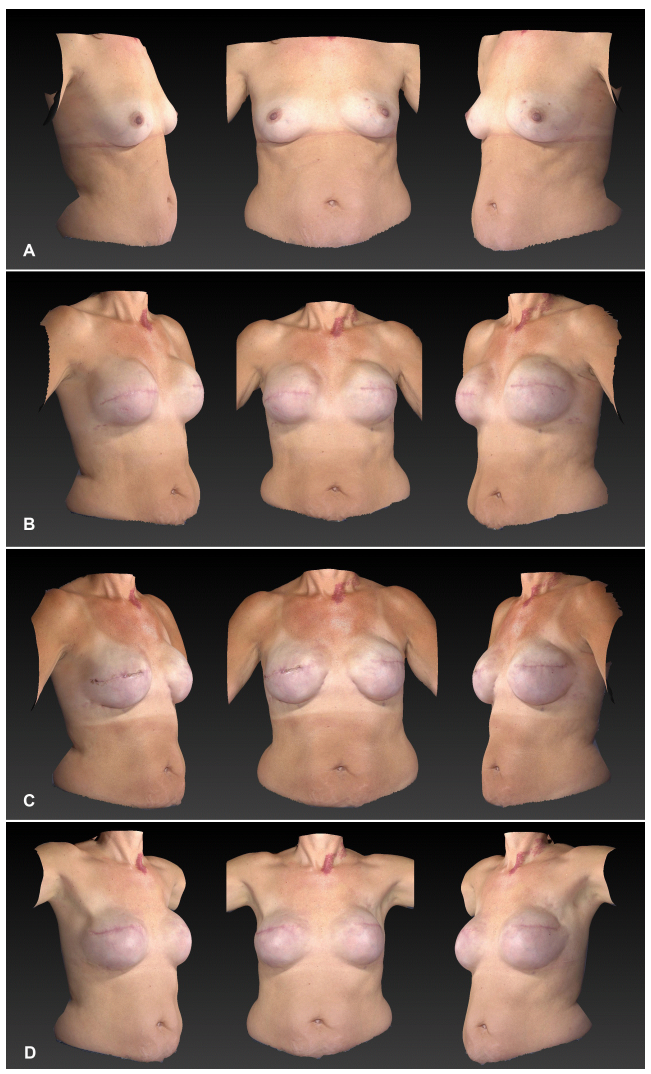

Figure 4: A 48 year old female with left breast ductal carcinoma in situ underwent bilateral simple mastectomies with and immediate tissue expander reconstruction. a) Preoperative imaging with a baseline breast volume of $278 \mathrm{~g}$ and $305 \mathrm{~g}$. b) Post-expansion imaging with tissue expanders filled to $450 \mathrm{cc}$ bilaterally. c) Imaging at 6 weeks post exchange of tissue expander for the permanent implant, with upsizing by $50 \mathrm{cc}$ to a volume of $500 \mathrm{cc}$ bilaterally. Inframammary fold reconstruction and bilateral upper pole fat grafting was completed at the same surgery. d) Imaging at 4 months post IMF reconstruction, demonstrating resolution of the dimpling seen along the IMF

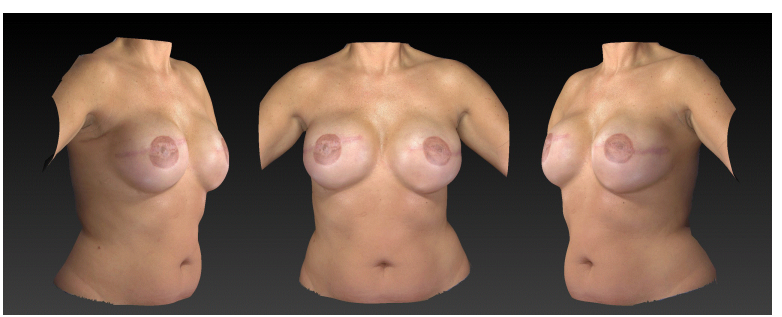

Figure 5: A 43 year old female with Stage 2A right breast infiltrating ductal carcinoma (IDC) underwent a right modified radical mastectomy with left prophylactic mastectomy and immediate tissue expander reconstruction. The patient desired increased volume and projection from her baseline breast volume of $350 \mathrm{~g}$ and $324 \mathrm{~g}$. Tissue expanders were filled to $480 \mathrm{cc}$, and the final implant volume was $550 \mathrm{cc}$ bilaterally. Images are taken 15 months after expander exchange and IMF reconstruction.

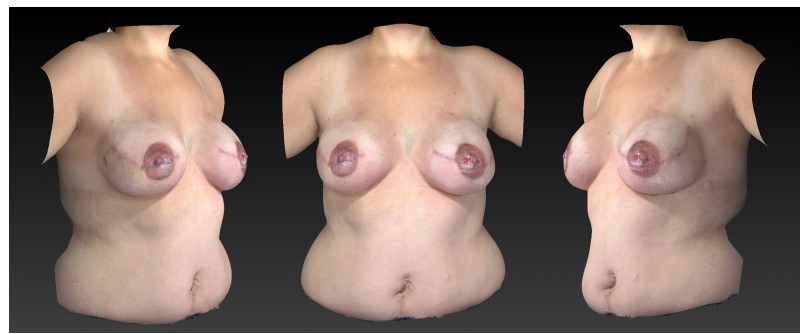

Figure 6: After positive BRCA testing, a 27 year old female underwent bilateral prophylactic mastectomies and immediate reconstruction. Tissue expanders were filled to $500 \mathrm{cc}$ bilaterally, and 550cc extra-high projection implants were placed. Two months later, the patient had bilaterally upper pole fat grafting with nipple reconstruction. Images are from 4 months after the IMF reconstruction.

As the breast develops, the IMF plays a crucial role to anchor the inferior pole of the breast to the chest wall and provide the classic shape to the breast itself [8]. However, in patients undergoing mastectomy, most if not all of the IMF is sacrificed during the oncologic resection and closure. Previous studies have documented only $0.7-1.7 \%$ of breast cancers arising in the IMF, however $28 \%$ of IMF specimens studied contained breast or lymphatic tissues, supporting the thought that the area should be resected in oncologic cases [9]. Despite the ongoing debate regarding IMF excision, this area commonly is destroyed during mastectomy and tissue expansion [10]. The IMF system can be challenging to recreate since histologic evaluation shows it is a dense collagen network in the dermis and not a simple ligamentous structure to mimic [11]. External bolsters attempt to create the IMF through scarring, but still lack true definition with ptosis, highlighting the need for a true reconstructive technique [12].

Early inframammary fold reconstruction was highlighted in 1977 by Pennisi, who used a dermal-superficial fascia flap anchored to the chest wall muscular fascia [13]. Although the external approaches were reliable, the overall fold was bulky and still did not allow for much ptosis. Current internal techniques are adapted from Handel's description in a small series, where an elevated capsulotomy is 
performed and the upper abdominal flap is fixed at the new IMF to increase the pocket and provide fold definition [14]. Nava has modified this to perform the capsulotomy at the desired level for increased precision, eliminated the dissection along the chest wall creating the upper abdominal lipofascial flap, and used a running suture between the superficial fascia and posterior capsule [13]. While this produced improved aesthetic results, there was still loss of definition in $5 \%$ of cases in the immediate postoperative period [13]. These procedures use an upper abdominal flap or superficial fascia sutured to the deep fascia, but this can lead to loss of fold definition. Thus, our technique anchors the dermis to the posterior capsule to create lasting definition and employs a silk suture to promote scarring and fixation.

While several factors aid in determining the aesthetics of the female breast, the inframammary fold is an important aspect. Few criteria exist regarding fold reconstruction, although a small series performed IMF re-suspension if the fold was undermined more than $1 \mathrm{~cm} \mathrm{[15].}$ Since the IMF can lose definition at a rate of $50 \%$ within 5 years, reconstructive surgeons should strive to redefine even acceptable folds [16]. Incorporating Acellular Dermal Matrix (ADM) can permit a more precise definition of the IMF, if the coverage is needed [17]. Therefore, achieving an improved aesthetic result hinges on the appearance of the inframammary and lateral mammary folds $[12,15]$.

Our procedure for inframammary fold reconstruction employs several techniques not previously used. First, applying capsulotomies along each edge of the breast allows for the skin release described by Bogetti. However, these are not carried out at the reflection to the posterior capsule but anteriorly into the breast in order to maintain implant placement without migration and improve anterior projection. Using the specific placement of the inferior capsulotomy to determine the fold's final position in addition to IMF re-suspension in the upright position has not been previously described; this important step allows for accurate placement of the IMF. Many authors then anchor the lower mastectomy dermis or subcutaneous fascia of the upper abdominal flap to the rib periostium $[3,15]$. While this stabilizes the lower pole, it can generate extreme and unnecessary postoperative discomfort. We have found that the early scalloping seen with dermalcapsular suspension resolves over the ensuing 6-8 weeks, and recovery is rapid with minimal to no discomfort.

The inframammary fold is an important aspect of an aesthetic reconstruction, yet it is not the only element needed to improve breast symmetry, projection, and shape. Placement of high and extra-high profile implants will assist in achieving upper pole fullness as we have found patient expectations tend towards that of an augmented breast. There is limited discussion in the literature regarding implant selection; however, surgeon variability persists. Although one author described using lower-profile, anatomic implants to minimize projection in the upper pole; still another author indicated high and extra-high profile implants as more optimal in breast reconstruction $[3,18]$. The BREAST-Q survey, a validated instrument to assess patient satisfaction, demonstrated higher patient satisfaction with silicone implants, since the newer silicone implants generate a more natural feel and less rippling with no discernable difference between anatomic and round implants [19]. In our practice, the majority of patients request increased anterior projection with upper pole fullness and prefer higher profile implants to achieve the desired outcome. We have attained this by upsizing the implant volume from the final tissue expander volume at the second-stage procedure. In addition, symmetry is confirmed during intraoperative evaluation of the patient in the sitting position during the reconstruction procedure.

Implant-based breast reconstruction is primarily comprised of single-stage and two-stage reconstructions, and several studies compare aesthetic outcomes and surgical revision rates. A prospective study comparing the two techniques noted a $70 \%$ reoperation rate in the single stage cohort when compared with the two-stage cohort (10\%) [20]. While patient satisfaction in the one-stage cohort was statically lower, this difference equalized after surgical revision of this cohort [20]. Bonomi et al. have found two-stage reconstructions achieve good results across a variety of patients, while single-stage reconstructions are best utilized in small, nonptotic breasts [21]. We have found staging affords an opportunity to apply these strategies in a stepwise and thoughtful manner and correlates with high patient satisfaction. In addition, we have found that two-stage reconstruction allows for increased patient involvement and a shared understanding of the patient's reconstructive goals during the expansion phase.

An additional strategy that has found new applications is the use of lipofilling in reconstructive surgery. Fat grafting can be applied to mastectomy flaps and the upper pole region to improve contour deformities, decrease implant rippling, reduce hollowing, and create a smooth transition from the chest wall to the implant. The timing of lipofilling can vary based on the desired objective. In a small case series, when structural fat grafting was applied to the mastectomy flaps of irradiated tissue prior to expander exchange, there were no reported cases of wound infection or ulceration [22]. Further studies have shown an aesthetic improvement when lipofilling is employed after permanent implant placement to correct reconstruction asymmetries $[23,24]$. In one study, patient aesthetic scores improved from 5.2 to 7.2 (scale 1-10), while surgeon scores increased from 4.9 to 7.1 at six months postoperatively [23]. We routinely employ lipofilling to mask implant visibility and correct rippling or contour defects. Additionally, we have found anecdotally that lipofilling tends to improve the quality of irradiated tissue and provides a softer pole. We have found lipofilling to be best tolerated when combined as an adjunctive procedure during the exchange procedure or nipple reconstruction.

Recreating the IMF through precise capsulotomy, abdominal flap recruitment/release, and suture suspension achieves improved breast positioning and definition against the chest wall with natural lower pole ptosis. Similarly, upsizing the implant at the second stage surgery allows the increased volume to adequately fill the expanded pocket and generate the desired upper pole fullness and anterior projection. This can be further enhanced with higher profile implants and use of lipofilling. While there are many different techniques to recreate the inframmary fold, we highlighted key elements from the original procedure as described by Pennisi, Nava and Bogetti. We do not discuss the muitlple minor modifications available to limit repetition in the manuscript. In regards to implant upsizing and the technique to mimic the augmented breast, this is the first paper to describe this procedure to our knowledge. As demonstrated in $2 \mathrm{D}$ and $3 \mathrm{D}$ photography, this technique highlights improved aesthetic outcomes mimicking the augmented breast. Further evaluation of patient satisfaction using the BREAST-Q survey, a validated instrument to assess patient satisfaction in breast reconstruction, is needed in this unique, urban population. 
Citation: Rodby KA, Quinn KP, Mehrara B and Antony AK (2014) Current Advances for Aesthetic Improvement in Breast Reconstruction: Mimicking the Augmented Breast . Surgery Curr Res 4: 202. doi:10.4172/2161-1076.1000202

Page 6 of 6

\section{Conclusion}

Current advances in two-stage implant based breast reconstruction are outlined to achieve upper pole fullness, anterior projection of the breast, and definition of the inframammary fold. . These results can be further enhanced with higher profile implants and the use of lipofilling, however further studies in patient satisfaction using the BREAST-Q survey are warranted. These strategies afford reconstructive outcomes that parallel the desired aesthetic features of the augmented breast.

\section{Conflicts/ disclosures}

A portion of this work was sponsored by the Warren and Clara Cole Foundation / American College of Surgeons Early Career Development Award, received by Dr. Anuja Antony. The remaining authors have no reportable conflicts or disclosures.

\section{References}

1. Avis NE, Crawford S, Manuel J (2004) Psychosocial problems among younger women with breast cancer. Psychooncology 13: 295-308.

2. Bogetti P, Cravero L, Spagnoli G, Devalle L, Boriani F, et al. (2007) Aesthetic role of the surgically rebuilt inframammary fold for implantbased breast reconstruction after mastectomy. Journal of Plastic, J Plast Reconstr Aesthet Surg 60: 1225-1232.

3. Panettiere P, Marchetti L, Accorsi D, Del Gaudio GA (2002) Aesthetic breast reconstruction. Aesthetic Plast Surg 26: 429-435.

4. Wilkins EG, Alderman AK (2004) Breast reconstruction practices in north america: current trends and future priorities. Semin Plast Surg 18 149-155.

5. Albornoz CR, Bach PB, Mehrara BJ, Disa JJ, Pusic AL, et al. (2013) A paradigm shift in U.S. Breast reconstruction: increasing implant rates. Plast Reconstr Surg 131: 15-23.

6. Pompei S, Frascino L, Marcasciano F, Caravelli G, Abate O, et al. (2012) Definition of the inframammary fold in breast reconstruction: A simplified option. Eur J Plast Surg 45: 723-729.

7. Ramon Y, Ullmann Y, Moscona R, Ofiram E, Tamir A, et al. (1997) Aesthetic results and patient satisfaction with immediate breast reconstruction using tissue expansion: a follow-up study. Plast Reconstr Surg 99: 686-691.

8. Muntan CD, Sundine MJ, Rink RD, Acland RD (2000) Inframammary fold: a histologic reappraisal. Plast Reconstr Surg 105: 549-556.

9. Gui GP, Behranwala KA, Abdullah N, Seet J, Osin P, et al. (2004) The inframammary fold: contents, clinical significance and implications for immediate breast reconstruction. Br J Plast Surg 57: 146-149.
10. Carlson GW, Grossl N, Lewis MM, Temple JR, Styblo TM (1996) Preservation of the inframammary fold: what are we leaving behind? Plast Reconstr Surg 98: 447-450.

11. Boutros S, Kattash M, Wienfeld A, Yuksel E, Baer S, et al. (1998) The intradermal anatomy of the inframammary fold. Plast Reconstr Surg 102: 1030-1033.

12. Chun YS, Pribaz JJ (2005) A simple guide to inframammary-fold reconstruction. Ann Plast Surg 55: 8-11.

13. Nava M, Quattrone P, Riggio E (1998) Focus on the breast fascial system: a new approach for inframammary fold reconstruction. Plast Reconstr Surg 102: 1034-1045.

14. Handel N, Jensen JA (1992) An improved technique for creation of the inframammary fold in silicone implant breast reconstruction. Plast Reconstr Surg 89: 558-562.

15. Topol B, Dalton E, Ponn T, Campbell C (2008) Immediate single-stage breast reconstruction using implants and human acellular dermal tissue matrix with adjustment of the lower pole of the breast to reduce unwanted lift. Ann Plast Surg 61: 494-499.

16. Persichetti P, Langella M, Filoni A, Cagli B, Tenna S (2013) How to redefine the inframammary fold: the "slingshot" capsular flap. Ann Plast Surg 70: 636-638.

17. Kim JY, Connor CM (2012) Focus on technique: two-stage implantbased breast reconstruction. Plast Reconstr Surg 130: 104S-15S.

18. Adams WP Jr (2013) Commentary on: clinical trial outcomes of highand extra high-profile breast implants. Aesthet Surg J 33: 540-543.

19. Macadam S, Ho A, Lennox P, Pusic A (2013) Patient-reported satisfaction and health-related quality of life following breast reconstruction: A comparison of shaped cohesive gel and round cohesive gel implant recipients. Plast Reconstr Surg 131: 431-441.

20. Eriksen C, Lindgren EN, Frisell J, Stark B (2012) A prospective randomized study comparing two different expander approaches in implant-based breast reconstruction: One stage versus two stages. Plast Reconstr Surg 130: 245-264.

21. Bonomi S, Settembrini F (2013) One-stage and two-stage approaches in implant-based breast reconstruction: in search of the best solution? Plast Reconstr Surg 131: 923e-924e.

22. Ribuffo D, Atzeni M, Guerra M, Bucher S, Politi C, et al. (2013) Treatment of irradiated expanders: Protective LIpofilling allows immediate prosthetic breast reconstruction in the setting of postoperative radiotherapy. Aesth Plast Surg 37: 1146-1152.

23. Cigna E, Ribuffo D, Sorvillo V, Atzeni M, Piperno A, et al. (2012) Secondary lipofilling after breast reconstruction with implants. Eur Rev Med Pharmacol Sci 16: 1729-1734.

24. Kanchwala SK, Glatt BS, Conant EF, Bucky LP (2009) Autologous fat grafting to the reconstructed breast: the management of acquired contour deformities. Plast Reconstr Surg 124: 409-418. 